\title{
Effect of cigarette smoking on plasma uric acid concentrations
}

\author{
Dhouha Haj Mouhamed • Asma Ezzaher • \\ Fadoua Neffati - Wahiba Douki • Lotfi Gaha • \\ Mohamed Fadhel Najjar
}

Received: 23 July 2010/Accepted: 11 November 2010/Published online: 18 December 2010

(C) The Japanese Society for Hygiene 2010

\begin{abstract}
Objectives The purpose of this study was to examine the effect of cigarette smoking on plasma uric acid concentration and to determine the correlation between this parameter and the biological tobacco markers, plasma thiocyanate and urinary cotinine.

Methods The initial study was conducted on 300 subjects; 138 of them were nonsmokers (62 men and 76 women) aged 14-72 years and 162 were current smokers (145 men and 17 women) aged 16-85 years. Uric acid, creatinine, and urinary cotinine were determined by the enzymatic colorimetric method and plasma thiocyanate by selective electrode.

Results Plasma uric acid concentration was significantly lower in smokers than in nonsmokers. A statistically significant negative correlation was noted between the smoking status parameters, including both the number of cigarettes smoked/day $\quad\left(F_{3-161}=12.063 ; \quad r=-0.9968\right.$; $p=0.0001)$ and the duration of smoking $\left(F_{3-161}=1.305\right.$; $r=-0.9406 ; p=0.0274)$, and the plasma uric acid. Among smokers, we noted a negative correlation between uric acid and both plasma thiocyanates $(r=-0.437$; $p<0.05)$ and urinary cotinine $(r=-0.580 ; p<0.05)$.

Conclusion After excluding the other factors affecting the uric acid levels, the significant low plasma uric acid in smokers was attributed to a reduction of the endogenous
\end{abstract}

D. Haj Mouhamed $(\bowtie) \cdot$ A. Ezzaher · F. Neffati · W. Douki ·

M. F. Najjar

Laboratory of Biochemistry-Toxicology,

University Hospital of Monastir, 5000 Monastir, Tunisia

e-mail: hajdhouha@yahoo.fr

L. Gaha

Psychiatry Department, University Hospital of Monastir, 5000 Monastir, Tunisia production as a result of the chronic exposure to cigarette smoke that is a significant source of oxidative stress. Therefore, it is recommended to stop or reduce smoking and to introduce plasma uric acid estimation as a routine test, since it is cheap and simple to reflect the antioxidant level.

Keywords Cigarette smoking - Urinary cotinine - Plasma thiocyanate $\cdot$ Uric acid $\cdot$ Oxidative stress

\section{Introduction}

Cigarette smoking is known to contribute to many diseases, including cancer, chronic obstructive pulmonary disease, stroke, cardiovascular diseases, and peptic ulcers $[1,2]$. Investigators have attempted to elucidate the mechanisms of the pathogenesis associated with cigarette smoking, but the conclusions were not consistent. A basic hypothesis is that free radicals cause oxidative damage to macromolecules such as lipids, proteins, and DNA. Therefore, these radicals play an important role in the pathogenesis of diseases [3]. In accordance with this theory, antioxidants are believed to play important roles in resisting damage from oxidative stress resulting from cigarette smoking. Several studies were conducted concerning the relationship between smoking and antioxidants, focusing on the differences of the effect of antioxidants between smokers and nonsmokers. However, the results were controversial.

As cigarette smoke contains superoxide and reactive nitrogen species that readily react with various biomolecules, it has been hypothesized that some of the adverse effects of smoking may result from the oxidative damage to the endothelial cells, which results in nitric oxide deficiency. Nitric oxide regulates the vascular tone and its 
deficiency accelerates the insufficiency of the coronary artery and vasoconstriction in different tissues. Therefore, the imbalance between oxidants and antioxidants may play an important role in the smoker [4]. In addition, cigarette smokers have increased inflammatory responses that further enhance their oxidative stress; in humans, uric acid is the most abundant aqueous antioxidant, accounting for up to $60 \%$ of serum free radical scavenging capacity, and it is an important intracellular free radical scavenger during metabolic stress, including smoking [5, 6]. The measurement of its serum level, therefore, reflects the antioxidant capacity [7]. Nicotine is the main substance that results in addiction to smoking. Because nicotine is a substance specific to cigarette smoking, it can be used as a marker for the number of cigarettes consumed [8]. Therefore, it allows to quantify the amount that a subject smokes. Nicotine metabolites, including cotinine, nicotine- $\mathrm{N}$-oxide, and a number of derivatives, offer a priority consideration. These are more accurate than other biomarkers such as carboxyhemoglobin or carbon monoxide, which are not predictors of the number of cigarettes smoked. In addition, such biomarkers may be present as a result of exposure to other environmental factors [8].

The aim of this study was to demonstrate the possible effect of smoking on plasma uric acid concentration and to determine the correlation between this parameter and the biological tobacco markers, plasma thiocyanate and urinary cotinine.

\section{Materials and methods}

Study design

\section{Population}

The initial study was conducted on 300 voluntary subjects; 138 of them were nonsmokers (62 men and 76 women) aged $35.6 \pm 16.0$ years and 162 were current smokers (145 men and 17 women) aged $38.0 \pm 17.5$ years. This study had been approved by the local ethical committee. Written informed consent was obtained from all voluntary adult participants and from the parents of minors.

\section{Samples}

For plasma uric acid, creatinine, and thiocyanates, venous blood was drawn in tubes containing lithium heparinate and it was immediately centrifuged. The plasma samples were stored at $-20^{\circ} \mathrm{C}$ until analysis. Urine samples were obtained from the smokers and nonsmokers. These samples were either used on the same day or frozen at $-20^{\circ} \mathrm{C}$ until they were required for analysis. All of the samples were analyzed for urinary cotinine.

\section{Methods}

\section{Smoking questionnaire}

Subject information and cigarette smoking outcome data were collected in a structured interview. The available data were limited to the classification of smoking to three categories: never, former, or current. The majority of current and former smokers were able to provide information on the number of cigarettes they smoked and the duration of smoking. All subjects were questioned concerning their medical history and sociodemographic characteristics, including age, gender, education, and employment.

\section{Laboratory analysis}

Uric acid, creatinine, and urinary cotinine levels were determined by the enzymatic colorimetric method on the Konelab $30^{\mathrm{TM}}$ analyzer (Thermo Electron Corporation). Cotinine was expressed as micrograms per micromol of creatinine in urine. $\mathrm{SCN}^{-}$levels were determined using selective electrodes (Ionometer SevenMulti S80 ${ }^{\mathrm{TM}}$, Mettler Toledo, Schwerzenbach, Switzerland) and expressed as milligrams per liter in plasma.

\section{Statistical analysis}

The results were expressed as means \pm standard deviation (SD). Standard descriptive statistics, correlation coefficients, and significance tests were calculated using the SPSS 17.0 program. Differences between mean values were evaluated by Student's $t$-test and between frequency values by the $\chi^{2}$ test. Comparisons between smokers and nonsmokers in uric acid levels were applied by analysis of variance (ANOVA) after adjustment to potential confounders' factors (factors of arthrosclerosis such as cholesterol, high-density lipoproteins [HDL] and low-density lipoproteins (LDL), alcohol drinking, creatinine, and body mass index [BMI]). A $p$-value of less than 0.05 was considered to represent a statistically significant difference between groups.

\section{Results}

We noted that plasma uric acid was significantly lower in smokers than in nonsmokers $(p=0.0003$, Table 1$)$. It was also significantly lower in smoking men than nonsmoking men $\left(203 \pm 100\right.$ vs. $\left.337 \pm 100 ; p<10^{-7}\right)$. The same 
Table 1 Variation of uric acid, creatinine, urea, $\mathrm{SCN}^{-}$, and urinary cotinine levels according smoking status

\begin{tabular}{llll}
\hline Parameters & Smokers $(n=162)$ & Nonsmokers $(n=138)$ & $p$ value \\
\hline Age $($ years $)$ & $38.0 \pm 17.5$ & $35.6 \pm 16$ & 0.172 \\
Sex ratio $(\mathrm{men} /$ women$)$ & $8.52(145 / 17)$ & $0.81(62 / 76)$ & $<10^{-3}$ \\
BMI $\left(\mathrm{kg} / \mathrm{m}^{2}\right)$ & $24.24 \pm 3.17$ & $25.63 \pm 4.36$ & 0.003 \\
Plasma creatinine $(\mu \mathrm{mol} / \mathrm{L})$ & $66.78 \pm 20.7$ & $72.3 \pm 19$ & 0.018 \\
Plasma uric acid $(\mu \mathrm{mol} / \mathrm{L})$ & $199 \pm 97$ & $250 \pm 132$ & 0.0003 \\
Plasma urea $(\mathrm{mmol} / \mathrm{L})$ & $4.0 \pm 1.9$ & $4.1 \pm 3.3$ & 0.05 \\
Urinary cotinine $(\mu \mathrm{g} / \mu \mathrm{mol} \mathrm{Cr})$ & $231.4 \pm 205.2$ & $73.2 \pm 73.7$ & $<10^{-7}$ \\
Plasma SCN $(\mu \mathrm{mol} / \mathrm{L})$ & $100.25 \pm 1.36$ & $99.62 \pm 0.91$ & 0.0005 \\
\hline
\end{tabular}

$\left(F_{3-161}=1.305 ; r=-0.9406 ; p=0.0274\right.$, Fig. $\left.2 b\right)$, and plasma uric acid.

Figures 3 and 4 show a negative correlation between urinary cotinine and plasma uric acid levels $(r=-0.580)$, and between plasma $\mathrm{SCN}^{-}$and uric acid concentrations $(r=-0.437)$.

\section{Discussion}

Many, but not all, epidemiological studies have suggested that high plasma uric acid is a risk factor for cardiovascular diseases, and they aimed at evaluating its prognostic implications and potential utility in the therapy monitoring $[9,10]$. This raised level of plasma uric acid, parallel to an increased risk of cardiovascular diseases, could be either primary or secondary to the underlying causes of the cardiovascular diseases [11]. However, the specific role of plasma uric acid in this constellation remains uncertain, although it may be involved in the platelet adhesiveness, aggregation, or inflammation, and it may be implicated in the genesis of hypertension [12]. In contrast, there is some evidence that the increase of plasma uric acid is protective against the cardiovascular diseases, since uric acid acts as an endogenous antioxidant $[12,13]$, and the higher plasma uric acid levels found in cardiovascular diseases patients suggest that any protective antioxidant effect of uric acid is hidden by other negative effects in these pathogeneses.

In this study, the plasma uric acid level in smokers was significantly lower than in nonsmokers $(p=0.0003)$, both in men $\left(p<10^{-7}\right)$ and in women $(p=0.02)$. This could confirm the effect of cigarette smoking on uric acid levels independently of the gender. In addition, we noted a significant negative correlation with the smoking status, including the average number of cigarettes smoked/day and the smoking duration. Moreover, we noted that the uric acid levels decrease when the smoking duration exceeds 5 years. This finding is in agreement with other studies showing a low plasma uric acid in regular smokers [14] and a reduction of antioxidants, including uric acid, in smokers, indicating that oxidative stress increases each time a 
Table 2 Variation of uric acid according to the number of cigarettes smoked and consumption duration

\begin{tabular}{|c|c|c|c|c|c|c|}
\hline & \multicolumn{2}{|c|}{ Cigarettes smoked/day } & \multirow[t]{2}{*}{$p$ value } & \multicolumn{2}{|c|}{ Consumption duration (years) } & \multirow[t]{2}{*}{$p$ value } \\
\hline & $<20$ & $\geq 20$ & & $\leq 5$ & $>5$ & \\
\hline \multicolumn{7}{|c|}{ Uric acid $<200 \mu \mathrm{mol} / \mathrm{L}$} \\
\hline $\mathrm{M}(n=64)$ & $141 \pm 25$ & $96 \pm 27$ & 0.004 & $140 \pm 41$ & $99 \pm 65$ & 0.01 \\
\hline $\mathrm{W}(n=8)$ & $185 \pm 17$ & $135 \pm 27$ & 0.03 & $175 \pm 20$ & $130 \pm 28$ & 0.04 \\
\hline \multicolumn{7}{|c|}{ Uric acid $>200 \mu \mathrm{mol} / \mathrm{L}$} \\
\hline $\mathrm{M}(n=81)$ & $269 \pm 42$ & $268 \pm 52$ & 0.96 & $271 \pm 50$ & $267 \pm 45$ & 0.69 \\
\hline $\mathrm{W}(n=9)$ & $276 \pm 72$ & $229 \pm 25$ & 0.38 & $304 \pm 78$ & $228 \pm 22$ & 0.39 \\
\hline$\chi^{2}, p$ & \multicolumn{3}{|c|}{$22.4 ; p<10^{-7}$} & \multicolumn{3}{|c|}{$3.89 ; p=0.04$} \\
\hline
\end{tabular}

$M$ men, $W$ women

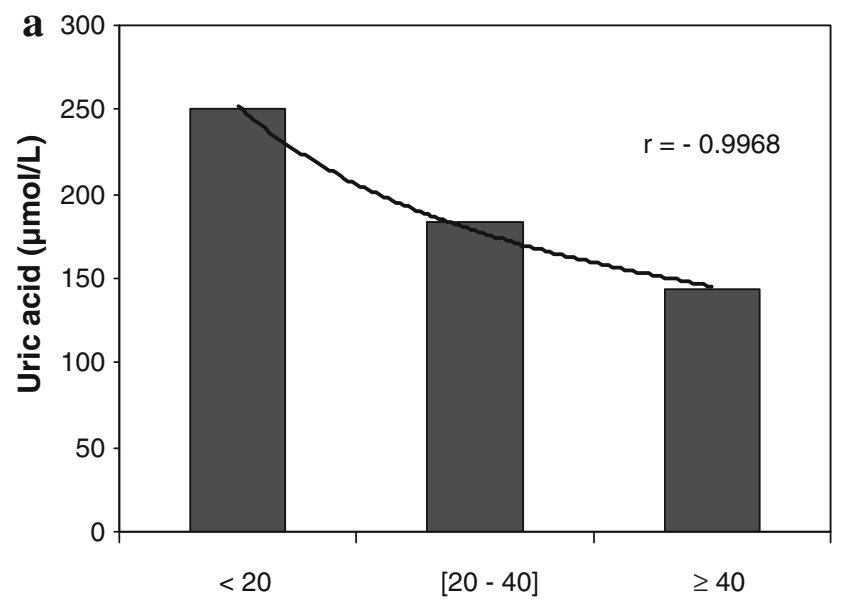

Cigarettes smoked/day

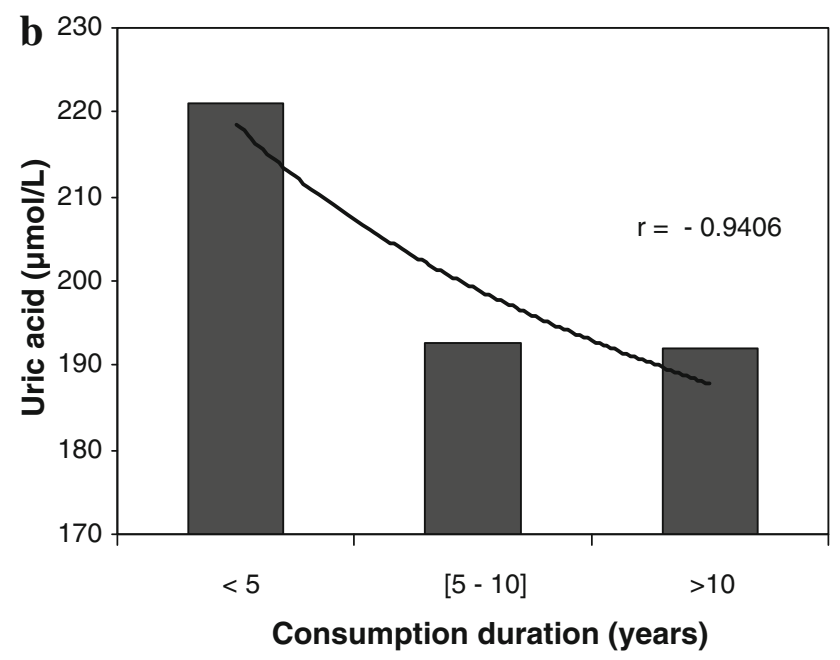

Fig. 2 Correlation between uric acid concentration and smoking status (cigarettes smoked/day and consumption duration). a Uric acid levels and cigarettes smoked/day. b Uric acid levels and consumption duration

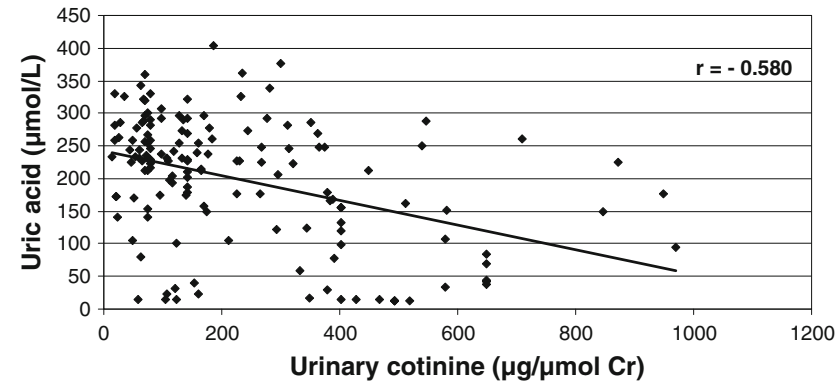

Fig. 3 Correlation between uric acid concentration and urinary cotinine levels

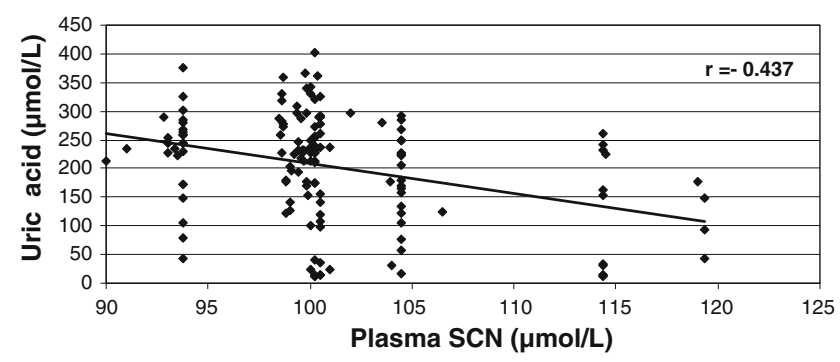

Fig. 4 Correlation between uric acid concentration and plasma $\mathrm{SCN}^{-}$levels

cigarette is smoked $[15,16]$. Other studies proved that even nonsmokers exposed to cigarette smoke have a significantly lower plasma antioxidant status than unexposed nonsmokers, independently of the differences in the dietary antioxidant intake [16]. Others studies proved that the administration of uric acid increases the circulating antioxidant defenses and allows the restoration of endothelium-dependent vasodilatation [17]. A decrease of uric acid in smokers can be explained by the inactivation of xanthine oxidase by cyanide, which is eliminated as thiocyanate $[18,19]$. Therefore, high plasma uric acid concentrations 
might be protective in situations characterized by an increase of cardiovascular risk and oxidative stress, such as smoking [14], and a reduction of its level, which increases susceptibility to oxidative damage and accounts for the excessive free radical production [16]. Therefore, the possibility that uric acid confers protection against the development of atherosclerosis, in view of its antioxidant properties, has been recognized [16].

In this study, we found a significant decrease of plasma creatinine levels in smokers compared to nonsmokers, although these values are not pathological. This can confirm that all of the subjects studied are without any renal failure, since the determination of creatinine has been reported to be useful in evaluating the renal handling of uric acid and as concentrations of this parameter are highly dependent on endogenous production as well as on renal excretion [19, 20]. Therefore, the low plasma uric acid level in smokers is attributed to a reduction of endogenous production.

This finding is in agreement with other studies that proved that the reduction of antioxidants, including uric acid in smokers, is due to both the chronic exposure to cigarette smoke, which is a significant source of oxidative stress, and to the low intake of dietary antioxidants [21].

Some of the relationships between tobacco and urea or uric acid are very significant; however, they are all very weak. If these relationships have the same origin, a hypothetical renal mechanism must first be considered. In fact, the blood urea is a product of the catabolism of proteins and their amino acids, whereas uric acid originates from the oxidation of purines. Moreover, the two molecules, while circulating in the blood, remain unlinked, either directly or by a common carrier. On the contrary, they are both excreted by the kidney, and in the disease processes, they generally vary in the same way: a rise in blood uric acid is well known as an early sign of renal failure. An increase in the renal excretion of urea and uric acid under the influence of tobacco is, therefore, a reasonable hypothesis, and it is supported by the known action of nicotine on the metabolism of catecholamines and the effect of these substances on renal function [22].

Urinary cotinine and plasma $\mathrm{SCN}^{-}$concentrations were both significantly higher in smokers than in nonsmokers, and they were well correlated with the number of cigarettes smoked per day and with the duration of consumption. Although cotinine and plasma $\mathrm{SCN}^{-}$are influenced by diet and industrial pollution, it remains a reliable indicator of the smoking status [23].

We found a negative correlation between the plasma uric acid level and both urinary cotinine concentration $(r=-0.580)$ and plasma $\mathrm{SCN}^{-}$concentration $(r=$ $-0.437)$ in active smokers. The important correlation found between urinary cotinine and plasma uric acid in smokers was not surprising because the urinary cotinine and plasma $\mathrm{SCN}^{-}$levels were determined as a marker of tobacco smoke exposure [24].

In our study, plasma antioxidant levels were closely, but inversely, related to the levels of plasma nicotine metabolites. It can be explained that more regular cigarette smoking will markedly affect plasma nicotine metabolites, and, thus, decrease plasma antioxidant levels. Furthermore, our finding suggests that plasma nicotine metabolites are appropriate as biomarkers for smoking consumption. These biomarkers should be applied in future studies on cigarette smoking.

\section{Limitations}

Several methodological limitations should be considered when interpreting these findings. First, larger sample sizes of the groups would be beneficial. Second, our work is a cross-sectional study that does not permit to follow up biological parameters. Finally, the sample of smokers may not be representative of more heterogeneous populations.

\section{Conclusion}

After the exclusion of other factors affecting uric acid levels, the significant low plasma uric acid level in smokers was attributed to a reduction of endogenous production as a result of the chronic exposure to cigarette smoke that is a significant source of oxidative stress. Therefore, cigarette smoking may influence oxidative stress by affecting the levels of plasma antioxidants, which may be involved in the mechanisms underlying various diseases. As this reduction is proportionate with the smoking status and leads to cardiovascular diseases, it is recommended to stop or reduce smoking and to introduce plasma uric acid estimation as a routine test, since it is cheap and simple to reflect the antioxidant status.

Acknowledgments The authors thank Mr. Samir Boukattaya for his assistance with the English language corrections.

Conflict of interest The authors stated that there are no conflicts of interest regarding the publication of this article.

\section{References}

1. Leistikow BN, Tsodikov A. Cancer death epidemics in United States Black males: evaluating courses, causation, and cures. Prev Med. 2005;41:380-5.

2. Mallampalli A, Guntupalli KK. Smoking and systemic disease. Clin Occup Environ Med. 2006;5:173-92.

3. Chiu YW, Chuang HY, Huang MC, Wu MT, Liu HW, Huang CT. Comparison of plasma antioxidant levels and related metabolic parameters between smokers and non-smokers. Kaohsiung J Med Sci. 2009;25:423-30. 
4. van der Vaart H, Postma DS, Timens W, ten Hacken NHT. Acute effects of cigarette smoke on inflammation and oxidative stress: a review. Thorax. 2004;59:713-21.

5. Mathru M, Dries DJ, Barnes L, Tonino P, Sukhani R, Rooney MW. Tourniquet-induced exsanguination in patients requiring lower limb surgery. An ischemia-reperfusion model of oxidant and antioxidant metabolism. Anesthesiology. 1996;84:14-22.

6. Hellsten Y, Tullson PC, Richter EA, Bangsbo J. Oxidation of urate in human skeletal muscle during exercise. Free Radic Biol Med. 1997;22:169-74.

7. Maxwell SR, Thomason H, Sandler D, LeGuen C, Baxter MA, Thorpe GH, et al. Antioxidant status in patients with uncomplicated insulin-dependent and non-insulin-dependent diabetes mellitus. Eur J Clin Invest. 1997;27:484-90.

8. Leone A. Biochemical markers of cardiovascular damage from tobacco smoke. Curr Pharm Des. 2005;11:2199-208.

9. Meisinger C, Koenig W, Baumert J, Döring A. Uric acid levels are associated with all-cause and cardiovascular disease mortality independent of systemic inflammation in men from the general population: the MONICA/KORA cohort study. Arterioscler Thromb Vasc Biol. 2008;28:1186-92.

10. Ioachimescu AG, Brennan DM, Hoar BM, Hazen SL, Hoogwerf BJ. Serum uric acid is an independent predictor of all-cause mortality in patients at high risk of cardiovascular disease: a preventive cardiology information system (PreCIS) database cohort study. Arthritis Rheum. 2008;58:623-30.

11. Kawamoto R, Tomita H, Oka Y, Ohtsuka N. Relationship between serum uric acid concentration, metabolic syndrome and carotid atherosclerosis. Intern Med. 2006;45:605-14.

12. Waring WS, Webb DJ, Maxwell SR. Systemic uric acid administration increases serum antioxidant capacity in healthy volunteers. J Cardiovasc Pharmacol. 2001;38:365-71.

13. Schretlen DJ, Inscore AB, Jinnah HA, Rao V, Gordon B, Pearlson GD. Serum uric acid and cognitive function in communitydwelling older adults. Neuropsychology. 2007;21:136-40.

14. Waring WS, Convery A, Mishra V, Shenkin A, Webb DJ, Maxwell SR. Uric acid reduces exercise-induced oxidative stress in healthy adults. Clin Sci. 2003;105:425-30.
15. Tsuchiya M, Asada A, Kasahara E, Sato EF, Shindo M, Inoue M. Smoking a single cigarette rapidly reduces combined concentrations of nitrate and nitrite and concentrations of antioxidants in plasma. Circulation. 2002;105:1155-7.

16. Dietrich M, Block G, Norkus EP, Hudes M, Traber MG, Cross $\mathrm{CE}$, et al. Smoking and exposure to environmental tobacco smoke decrease some plasma antioxidants and increase gammatocopherol in vivo after adjustment for dietary antioxidant intakes. Am J Clin Nutr. 2003;77:160-6.

17. Waring WS, McKnight JA, Webb DJ, Maxwell SRJ. Uric acid restores endothelial function in patients with type 1 diabetes and regular smokers. Diabetes. 2006;55:3127-32.

18. Massey V, Edmondson D. On the mechanism of inactivation of xanthine oxidase by cyanide. J Biol Chem. 1970;245:6595-8.

19. Puig JG, Mateos FA, Jiménez ML, Ramos TH. Renal excretion of hypoxanthine and xanthine in primary gout. Am J Med. 1988;85:533-7.

20. Leyva F, Wingrove CS, Godsland IF, Stevenson JC. The glycolytic pathway to coronary heart disease: a hypothesis. Metabolism. 1998;47:657-62.

21. Bloomer RJ. Decreased blood antioxidant capacity and increased lipid peroxidation in young cigarette smokers compared to nonsmokers: impact of dietary intake. Nutr J. 2007;6:39-40.

22. Watts DT. The effect of nicotine and smoking on the secretion of epinephrine. Ann N Y Acad Sci. 1960;90:74-90.

23. Bruckert E, Jacob N, Lamaire L, Truffert J, Percheron F, de Gennes JL. Relationship between smoking status and serum lipids in a hyperlipidemic population and analysis of possible confounding factors. Clin Chem. 1992;38:1698-705.

24. Haj Mouhamed D, Ezzaher A, Neffati F, Douki W, Najjar MF. Comparison between plasma and urine thiocyanates and urinary cotinine determinations as indicators of cigarette smoking. J Health Sci. 2009;55:1-6. 